\title{
Global Production Planning Process considering the Supply Risk of Overseas Manufacturing Sites
}

\author{
Hosang Jung ${ }^{1}$ and Seungbae Sim ${ }^{2}$ \\ ${ }^{1}$ Asia Pacific School of Logistics, Inha University, 100 Inha-ro, Nam-gu, Incheon 402-751, Republic of Korea \\ ${ }^{2}$ Korea Institute for Defense Analyses, 37 Hoegi-ro, Dongdaemun-gu, Seoul 130-871, Republic of Korea \\ Correspondence should be addressed to Hosang Jung; hjung@inha.ac.kr
}

Received 24 May 2015; Revised 16 July 2015; Accepted 6 August 2015

Academic Editor: Davide La Torre

Copyright (c) $2015 \mathrm{H}$. Jung and S. Sim. This is an open access article distributed under the Creative Commons Attribution License, which permits unrestricted use, distribution, and reproduction in any medium, provided the original work is properly cited.

\begin{abstract}
Although global manufacturers can produce most of their final products in local plants, they need to source components or parts from desirable overseas manufacturing partners at low cost in order to fulfill customer orders. In this global manufacturing environment, capacity information for planning is usually imprecise owing to the various risks of overseas plants (e.g., foreign governments' policies and labor stability). It is therefore not easy for decision-makers to generate a global production plan showing the production amounts at local plants and overseas manufacturing facilities operated by manufacturing partners. In this paper, we present a new global production planning process considering the supply risk of overseas manufacturing sites. First, local experts estimate the supply capacity of an overseas plant using their judgment to determine when the risk could occur and how large the risk impact would be. Next, we run a global production planning model with the estimated supply capacities. The proposed process systematically adopts the qualitative judgments of local experts in the global production planning process and thus can provide companies with a realistic global production plan. We demonstrate the applicability of the proposed process with a real world case.
\end{abstract}

\section{Introduction}

In recent years, most global manufacturers have pursued distributed manufacturing by cooperating with manufacturing partners to increase responsiveness to customer demand and avoid lost sales. However, global production environments are naturally uncertain, since various unexpected events can occur at overseas manufacturing sites. Machine breakdown, maintenance, labor strikes, and political instability are some of the major risks when trying to generate global production plans for upcoming planning periods. Moreover, collaboration with foreign manufacturing partners makes the global production environment more complex and uncertain, since it is more difficult for the manufacturer to obtain precise information about foreign manufacturing partners than to gather information from inside the company.

In this uncertain global production environment, global production planning (GPP) considering both local plants owned by the manufacturer and global manufacturing sites operated by manufacturing partners located in foreign countries has become an important issue.

A quick, rough estimation of the production capacities of foreign manufacturing sites for the upcoming planning periods is required to generate a global production plan. How then can a manufacturer estimate the supply capacity of overseas manufacturing sites? Among the various supply risks mentioned above, machine breakdown and maintenance occur regularly and thus can be considered in GPP by adopting a probabilistic approach. However, other risks also exist which are not well captured using the probabilistic approach. Foreign governments' policies, labor strikes, and political instability are representative risks that cannot be easily specified in advance. Thus, local experts who can better anticipate upcoming risks might be able to provide their opinions to the manufacturer when generating the global production plan. In fact, this research is motivated by a real world footwear supply chain, which will be presented in a later section. Practitioners' requirements are twofold: (1) to develop 
a reasonable way to estimate the supply capacity of foreign production lines considering the above-mentioned risks and (2) to reflect the opinions of local experts in such an estimation.

In this paper, we propose a new GPP process considering the supply risks of overseas manufacturing sites. In the proposed planning process, first, local experts estimate the supply capacity of the overseas plant using their judgment of when the risk could occur and how big the risk's impact would be. Second, we run a GPP model with the estimated supply capacities.

The paper is organized as follows. Related research works are presented in Section 2, and the proposed GPP model and related planning procedure are introduced in Section 3. In Section 4, a case study based on a real world industry is presented to show how the proposed process can be utilized. Finally, Section 5 suggests some future research directions with concluding remarks.

\section{Related Research}

2.1. Global Production Planning. For production planning models considering global supply chain network, a number of researches are investigated. Tsiakis and Papageorgiou [1] proposed a static mixed integer linear programming (MILP) model to formulate a strategic optimal planning problem for multiechelon supply chain networks with some financial considerations. Liu and Papageorgiou [2] developed a multiobjective MILP model for production, distribution, and capacity planning of global supply chains. They considered cost, responsiveness, and customer service level simultaneously, while many researchers only considered single criterion for the global production planning problems. MartínezCosta et al. [3] reviewed strategic capacity planning models and analyzed the objective function and constraints. Additionally, they classified mathematical models according to the characteristics of the model and the solution procedures. Lanza and Moser [4] described an approach for the strategic planning of global changeable production networks. The proposed approach is based on scenario forecasting for global production networks and a multiobjective optimization to identify the optimal network configuration. Shah and Ierapetritou [5] addressed the integrated planning and scheduling problem for the multisite, multiproduct batch plant. To solve the multisite production and distribution optimization problem, they applied augmented Lagrangian relaxation method. Meanwhile, Díaz-Madroñero et al. [6] reviewed tactical optimization models for integrated production and transport routing planning. They focused on the simultaneous consideration of production and transportation activities in globalized supply chains.

In recent years, managing supply and demand uncertainty in a global supply chain network has received much attention. Jabbarzadeh et al. [7] proposed a realistic production-distribution planning model with the robustness to common supply interruptions and demand variations. From numerical results, they examined the benefits of consideration of both supply and demand uncertainties and investigated the price of robustness under various supply and demand scenarios. Sahling and Kayser [8] addressed a stochastic supply network planning model with vendor selection. In this model, the supply chain configuration is determined by using long-term demand forecasting. They proposed a two-stage stochastic programming approach and numerical results showed a robust and stable supply chain network configuration under demand uncertainty. Most of the recent research focused on demand variations or uncertainty and did not consider the uncertainty for production capacity of overseas manufacturing sites.

2.2. Supply Chain Risks. Supply chain risks have been widely investigated for the past decade, as several supply chain disruptions such as natural disasters and the global financial crisis occurred [9-11]. Unpredictable supply chain risks can cause undesirable operational and financial impact [12]. Thus, many researchers have focused on supply chain risk management. Tang [13] reviewed various quantitative models for managing supply chain risks and classified them into supply management and demand management. For example, supply risks can be caused by uncertain supply yields, uncertain lead time, uncertain supply capacity, and so on. Demand risks can be caused by shifting demand across time, markets, and products. Ritchie and Brindley [14] explored the interaction between risk and performance in a supply chain context, presented a new framework to integrate the dimensions of risk and performance in supply chains, and analyzed risk drivers. Tang and Tomlin [15] addressed the importance of supply chain flexibility for mitigating supply chain risks and presented a unified framework and five models to show that firms can mitigate supply chain risks with a relatively low level of flexibility. Chopra and Sodhi [16] defined supply chain risks and their drivers and presented various strategies for mitigating those risks. Oke and Gopalakrishnan [17] categorized risks into inherent/high-frequency risks and disruptive/ infrequent risks and identified generic risk mitigation strategies.

Supply chain risks that can affect global manufacturing can be categorized as follows: operational risks, macro risks, and policy risks [10]. Among operational risks, risks from the machine or system can be taken into account by analyzing the historical process/system data and calculating various probabilities regarding process/system failure/breakdown. However, operational risks from human labor are more difficult to predict in terms of timing and impact. Furthermore, since both macro and policy risks (i.e., exogenous risks) are naturally uncertain, they cannot be fully estimated by any quantitative approach. Thus, the judgments of local experts could be used to get a rough estimation of how to manage such risks (i.e., labor-related operational risks, macro risks, and policy risks) in planning.

Even if there were lots of researches dealing with the quantitative models and methods for supply risk assessment, most of them have focused on the decision-making problem itself under supply risk environments. Thus, it seems that the risks in those researches were assumed to be probabilistic considering historical data or to be any predetermined 
scenario. Recently, the extensive review on supply chain risk focusing on definition, measure, and modelling was conducted by Heckmann et al. [18]. According to their review paper, one of the most important but not explored research topics regarding supply chain risk is to consider the attitude of the decision-makers. Supply chain risk, as risk in general, may be regarded as a subjective concept that relies on the individual's assessment of potential outcomes rather than an objective concept [19]. Thus, decision-makers' preferences have a decisive influence on the measurement of future supply chain performances and decisions [18]. In this paper, we focused on how the local experts judge the upcoming risks and tried to provide a systematic approach to reflect their opinions on the supply capacity estimation. Unlike the previous related researches, the proposed approach in this paper can be a good alternative to directly consider the attitude or preference of the decision-makers when judging the potential risks.

2.3. Managing Risks in Supply Chain Planning. For the past decade, many researchers have tried to incorporate supply chain risks in many supply chain planning problems. To manage supply chain risks, two representative approaches were adopted: stochastic programming and fuzzy mathematical programming. In general, stochastic programming approaches that involve risks are particularly suited for problems, where the risk-related data evolve over time and decisions have to be made prior to observing the entire data stream. Among the various risks, machine/system-related operational risks can be best handled by stochastic programming approaches. In practice, it is common to use scenarios to consider the expected risks in stochastic programming models [20]. Applications of stochastic programming for multisite production planning or supply chain planning can be found in Leung et al. [21], Zhang et al. [22], Karabuk [23], and so on. In fuzzy mathematical programming, some of the parameters and constraints are set to be fuzzy numbers and fuzzy equations, respectively. Some researchers have tried to apply the fuzzy mathematical programming to supply chain planning problems [24-26]. Each approach has pros and cons. If there is enough historical data regarding supply chain risks, stochastic approaches can be used to manage upcoming risks, as mentioned above. However, when historical data are unreliable or unavailable, this may not be the best choice [27, 28]. Compared with stochastic approaches, fuzzy approaches provide greater computational efficiency and flexibility in fuzzy arithmetic operations [29].

In this paper, we propose a new approach consisting of two steps: (1) estimating supply risks in terms of timing and impact and (2) generating a global production plan using a mixed integer linear programming (MILP) model. In the first step, we adopt fuzzy theory to convert the experts' qualitative judgments of the supply risks into the estimated supply capacity. Owing to the characteristics of the risk handled in this paper, fuzzy theory is adopted instead of stochastic approaches. Furthermore, the MILP model is used to generate the global production plan once the supply capacities are estimated.

\section{The GPP Process}

3.1. Model. We propose a GPP model. The basic structure of the model is based on the real world case presented in Section 4.

To formulate the mathematical model, the following assumptions are required:

(i) The supply chain is composed of a foreign supplier (i.e., original equipment manufacturer), a manufacturer, a distribution center, and a market.

(ii) Products are moved from the manufacturer to the markets via the distribution centers.

(iii) Demand is forecasted and known to a decisionmaker.

(iv) Unit production cost, setup cost, and inventory holding cost are precise and vary across locations, periods, and product types.

(v) Production capacity of the foreign suppliers is imprecise owing to the supply risk.

(vi) All the costs, product prices, and capacities are known to the decision-maker.

(vii) Planning is done for the upcoming several months, and the basic time unit is a month.

(viii) Production lead time is negligible owing to large time bucket (i.e., month) problem characteristics.

Furthermore, the following indices, parameters, and decision variables are required to formulate the model:

Indices

$$
\begin{aligned}
& f=1, \ldots, F \text { : foreign supplier's production facility, } \\
& a=1, \ldots, A \text { : manufacturer's assembly facility, } \\
& d=1, \ldots, D \text { : distribution center, } \\
& c=1, \ldots, C \text { : customer, } \\
& m=1, \ldots, M \text { : component (each product has several } \\
& \text { components), } \\
& k=1, \ldots, K: \text { product, } \\
& v=1, \ldots, V: \text { transportation vehicle, } \\
& t=1, \ldots, T \text { : time period (month). }
\end{aligned}
$$

\section{Parameters}

$p_{k c t}$ : market price for one unit of product $k$ sold to customer $c$ during period $t$,

$r_{m f t}^{1}:$ unit production cost for component $m$ in production facility $f$ during period $t$,

$r_{\text {kat }}^{2}$ : unit production cost for product $k$ in assembly facility $a$ during period $t$,

$u_{m f t}^{1}$ : production setup cost for component $m$ in production facility $f$ during period $t$,

$u_{k a t}^{2}:$ production setup cost for product $k$ in assembly facility $a$ during period $t$, 
$t_{m f a v t}^{1}$ : cost of transporting one unit of component $m$ from production facility $f$ to assembly facility $a$ using vehicle $v$ during period $t$,

$t_{k a d v t}^{2}$ : cost of transporting one unit of product $k$ from assembly facility $a$ to distribution center $d$ using vehicle $v$ during period $t$,

$t_{k d c v t}^{3}$ : cost of transporting one unit of product $k$ from distribution center $d$ to customer $c$ using vehicle $v$ during period $t$,

$h_{m f t}^{1}$ : unit inventory holding cost for component $m$ in production facility $f$ during period $t$,

$h_{\text {mat }}^{2}$ : unit inventory holding cost for component $m$ in assembly facility $a$ during period $t$,

$h_{k a t}^{2}$ : unit inventory holding cost for product $k$ in assembly facility $a$ during period $t$,

$h_{k d t}^{3}$ : unit inventory holding cost for product $k$ in distribution center $d$ during period $t$,

$H_{f t}^{1}$ : inventory capacity of production facility $f$ during period $t$,

$H_{a t}^{2}$ : inventory capacity of assembly facility $a$ during period $t$,

$H_{d t}^{3}$ : inventory capacity of distribution center $d$ during period $t$,

$s_{m}$ : storage space required for one unit of component $m$,

$s_{k}$ : storage space required for one unit of product $k$,

$c_{m f}:$ production capacity required to produce one unit of component $m$ in production facility $f$,

$c_{k a}$ : production capacity required to produce one unit of product $k$ in assembly facility $a$,

$C_{f t}$ : production capacity of production facility $f$ during period $t$,

$C_{a t}$ : production capacity of assembly facility $a$ during period $t$,

$T_{f a t}^{1}$ : transportation capacity from production facility $f$ to assembly facility $a$ during period $t$,

$T_{a d t}^{2}$ : transportation capacity from assembly facility $a$ to distribution center $d$ during period $t$,
$T_{d c t}^{3}:$ transportation capacity from distribution center $d$ to customer $c$ during period $t$,

$l_{\text {fav }}^{1}$ : transportation time (months) from production facility $f$ to assembly facility $a$ using vehicle $v$,

$l_{a d v}^{2}$ : transportation time (months) from assembly facility $a$ to distribution center $d$ using vehicle $v$,

$l_{d c v}^{3}$ : transportation time (months) from distribution center $d$ to customer $c$ using vehicle $v$,

$D_{k c t}$ : demand for product $k$ by customer $c$ during period $t$,

$b_{m k}$ : amount of component $m$ required to produce one unit of product $k$.

\section{Decision Variables}

$x_{m f t}$ : amount of component $m$ produced in production facility $f$ during period $t$,

$x_{k a t}$ : amount of product $k$ produced in assembly facility $a$ during period $t$,

$y_{m f a v t}^{1}$ : amount of component $m$ transported from production facility $f$ to assembly facility $a$ using vehicle $v$ during period $t$,

$y_{k a d v t}^{2}$ : amount of product $k$ transported from assembly facility $a$ to distribution center $d$ using vehicle $v$ during period $t$,

$y_{k d c v t}^{3}$ : amount of product $k$ transported from distribution center $d$ to customer $c$ using vehicle $v$ during period $t$,

$I_{m f t}^{1}$ : inventory amount of component $m$ in production facility $f$ during period $t$,

$I_{\text {mat }}^{2}$ : inventory amount of component $m$ in assembly facility $a$ during period $t$,

$I_{k a t}^{2}$ : inventory amount of product $k$ in assembly facility $a$ during period $t$,

$I_{k d t}^{3}$ : inventory amount of product $k$ in distribution center $d$ during period $t$,

$z_{m f t}$ : binary variable to capture the setup cost of component $m$ produced in production facility $f$ during period $t$,

$z_{k a t}$ : binary variable to capture the setup cost of product $k$ produced in assembly facility $a$ during period $t$.

The GPP model is expressed as follows:

$$
\begin{aligned}
\operatorname{Max} & \sum_{k=1}^{K} \sum_{c=1}^{C} \sum_{t=1}^{T}\left(p_{k c t} \sum_{d=1}^{D} \sum_{v=1}^{V} y_{k d c v t}^{3}\right)-\left\{\sum_{m=1}^{M} \sum_{f=1}^{F} \sum_{t=1}^{T}\left(r_{m f t}^{1} x_{m f t}+u_{m f t}^{1} z_{m f t}+h_{m f t}^{1} I_{m f t}^{1}\right)\right. \\
& +\sum_{k=1}^{K} \sum_{a=1}^{A} \sum_{t=1}^{T}\left(r_{k a t}^{2} x_{k a t}+u_{k a t}^{2} z_{k a t}+h_{k a t}^{2} I_{k a t}^{2}\right)+\sum_{m=1}^{M} \sum_{a=1}^{A} \sum_{t=1}^{T} h_{m a t}^{2} I_{m a t}^{2}+\sum_{k=1}^{K} \sum_{d=1}^{D} \sum_{t=1}^{T} h_{k d t}^{3} I_{k d t}^{3}
\end{aligned}
$$




$$
\left.+\sum_{m=1}^{M} \sum_{f=1}^{F} \sum_{a=1}^{A} \sum_{v=1}^{V} \sum_{t=1}^{T} t_{m f a v t}^{1} y_{m f a v t}^{1}+\sum_{k=1}^{K} \sum_{a=1}^{A} \sum_{d=1}^{D} \sum_{v=1}^{V} \sum_{t=1}^{T} t_{k a d v t}^{2} y_{k a d v t}^{2}+\sum_{k=1}^{K} \sum_{d=1}^{D} \sum_{c=1}^{C} \sum_{v=1}^{V} \sum_{t=1}^{T} t_{k d c v t}^{3} y_{k d c v t}^{3}\right\}
$$

subject to $I_{m f t}^{1}=I_{m f t-1}^{1}+x_{m f t}-\sum_{a=1}^{A} \sum_{v=1}^{V} y_{m f a v t}^{1} \quad \forall m, f, t$

$I_{m a t}^{2}=I_{m a t-1}^{2}-\sum_{k=1}^{K} b_{m k} x_{k a t}+\sum_{f=1}^{F} \sum_{v=1}^{V} y_{m f a d v t-l_{f a v}^{1}}^{1} \quad \forall k, a, t$

$I_{k a t}^{2}=I_{k a t-1}^{2}+x_{k a t}-\sum_{d=1}^{D} \sum_{v=1}^{V} y_{k a d v t}^{2} \quad \forall k, a, t$

$I_{k d t}^{3}=I_{k d t-1}^{3}+\sum_{a=1}^{A} \sum_{v=1}^{V} y_{k a d v t-l_{a d v}^{2}}^{2}-\sum_{c=1}^{C} \sum_{v=1}^{V} y_{k d c v t}^{3} \quad \forall k, d, t$

$\sum_{d=1}^{D} \sum_{v=1}^{V} y_{k d c v t-l_{d c v}^{3}}^{3} \leq D_{k c t} \quad \forall k, c, t$

$\sum_{m=1}^{M} c_{m f} x_{m f t} \leq C_{f t} \quad \forall f, t$

$\sum_{k=1}^{K} c_{k a} x_{k a t} \leq C_{a t} \quad \forall a, t$

$x_{m f t} \leq M \cdot z_{m f t} \quad \forall m, f, t$

$x_{k a t} \leq M \cdot z_{k a t} \quad \forall k, a, t$

$\sum_{m=1}^{M} s_{m} I_{m f t}^{1} \leq H_{f t}^{1} \quad \forall f, t$

$\sum_{k=1}^{K}\left(s_{m} I_{m a t}^{2}+s_{k} I_{k a t}^{2}\right) \leq H_{a t}^{2} \quad \forall a, t$

$\sum_{k=1}^{K} s_{k} I_{k d t}^{3} \leq H_{d t}^{3} \quad \forall d, t$

$\sum_{m=1}^{M} \sum_{v=1}^{V} s_{m} y_{m f a v t}^{1} \leq T_{f a t}^{1} \quad \forall f, a, t$

$\sum_{k=1}^{K} \sum_{v=1}^{V} s_{k} y_{k a d v t}^{2} \leq T_{a d t}^{2} \quad \forall a, d, t$

$\sum_{k=1}^{K} \sum_{v=1}^{V} s_{k} y_{k d c v t}^{3} \leq T_{d c t}^{3} \quad \forall d, c, t$

$I_{m f 0}^{1}=0$,

$I_{m a 0}^{2}=0$,

$I_{k a 0}^{2}=0$,

$I_{k d 0}^{3}=0$

$\forall m, k, f, a, d$ 


$$
\begin{aligned}
& x_{m f t}, x_{k a t}, I_{m f t}^{1}, I_{m a t}^{2}, I_{k a t}^{2}, I_{k d t}^{3}, y_{m f a v t}^{1}, y_{k a d v t}^{2}, y_{k d c v t}^{3} \geq 0 \quad \forall m, k, f, a, d, c, v, t \\
& z_{m f t}, z_{k a t} \in\{0,1\} \quad \forall m, k, f, a, t .
\end{aligned}
$$

The objective function (1) works to maximize total profit (sales revenue minus total costs). Constraint (2) models the inventory balances of components at production facilities considering the departing amounts towards assembly facilities. Constraint (3) models the inventory balances of components at assembly facilities considering the incoming amounts from production facilities and the production amounts of products. Constraint (4) models the inventory balances of products at assembly facilities considering the departing amounts towards distribution centers. Constraint (5) is also the inventory balance equation at the distribution centers considering both incoming and departing amounts. Constraint (6) limits the transportation amount from the delivery centers to customers to no more than the demand. Constraints (7) and (8) ensure that production capacity is not violated. Constraints (9) and (10) guarantee that the setup cost is incurred when production starts. Constraints (11), (12), and (13) address the inventory capacities at the production facilities, assembly facilities, and distribution centers, respectively. Constraints (14), (15), and (16) ensure transportation capacity. Constraint (17) indicates that the initial inventories are assumed to be zero. Finally, Constraint (18) is a nonnegativity constraint, and Constraint (19) defines the binary decision variables regarding production setup.

3.2. Supply Capacity Estimation Process. In this paper, to estimate the supply capacities of global manufacturing sites considering risks, we adopt a group decision-making approach that collects and merges the opinions of various supply chain experts who can predict and judge the upcoming risks of those global manufacturing sites. Compared with a traditional single decision-making process involving only a single decision-maker at the headquarters, this group decisionmaking approach might provide a clearer estimation of supply capacities because local experts better understand the societal, political, and economic risks of the foreign countries.

Thus, we newly propose an opinion collecting and merging process for estimating the supply capacity of any foreign manufacturing site considering risks. Two factors are used for estimating the supply capacity: (1) the timing of risk, that is, when the risk will occur during the given month, and (2) the impact of risk, that is, how big that risk will become.

The process of estimating supply capacity using the aforementioned two factors can be depicted as in Figure 1.

In Figure 1, the final supply capacity of any foreign manufacturing facility for any month (i.e., the basic time unit) is estimated based on how both factors are set.

In general, supply capacity is assumed to be given as one of the parameters of the production planning mathematical model when generating a production plan. However, in the real world, estimation usually includes a high degree of fuzziness and uncertainty [30]. Furthermore, supply chain experts very naturally provide uncertain answers rather than precise values, and it is difficult to transform qualitative preferences into point estimates [31].

Thus, we try to estimate supply capacity by asking local supply chain experts two questions regarding the abovementioned factors. The experts are asked to provide their opinion (answers) using the fuzzy approach instead of giving an exact value. For example, an expert can express his or her opinion on the impact of risk as the likelihood that the risk will have a very large impact on production capacity. In the proposed fuzzy approach, linguistic values expressed in triangular fuzzy numbers are used for simplicity and efficiency of computation. Triangular fuzzy numbers are convenient concept for adequately representing and arithmetically manipulating imprecise numerical quantities and subjective preference of decision-makers like our case in various decision-making situations [32]. Triangular fuzzy numbers have been widely used and validated in many applications for solving practical problems of various kinds in real world setting [32-34].

A triangular fuzzy number $\tilde{a}$ can be defined by the closed interval $[l, u]$ including its mean $m$ as follows:

$$
\mu_{\widetilde{a}}(x)= \begin{cases}\frac{x-l}{m-l}, & l \leq x \leq m, \\ \frac{u-x}{u-m}, & m \leq x \leq u \\ 0, & \text { otherwise }\end{cases}
$$

For the first question on the timing of risk, the following triangular fuzzy numbers are used to express the experts' answers: an expert could answer a question such as when the risk will occur during the given month using the linguistic expressions in Table 1.

Of course, if the expert expects no risk, he or she simply answers " 4 " to this question. From Table 1 and Figure 2, " 4 " indicates that risk will not occur during the given month.

The membership function corresponding to Table 1 is shown in Figure 2. If the number of supply chain experts providing opinions on the timing of risk is $K$ (this means that the expert pool consists of $K$ supply chain experts), we can obtain $K$ different opinions represented as positive triangular fuzzy numbers. Thus, we need to merge those fuzzy numbers into one integrated fuzzy number, $T$. Let us assume that $l_{k}^{T}$ is the lower value of the triangular fuzzy number representing the timing of risk, $m_{k}^{T}$ is the mean value of the triangular fuzzy number representing the timing of risk, and $u_{k}^{T}$ is the upper value of the triangular fuzzy number representing the timing of risk. Then, the integrated fuzzy number $\widetilde{T}$ can be obtained from the following equation:

$$
\widetilde{T}=\left(L^{T}, M^{T}, U^{T}\right)
$$


TABLE 1: Fuzzy numbers regarding the timing of risk.

\begin{tabular}{lc}
\hline Linguistic expression of opinion & Triangular fuzzy number \\
\hline At the beginning of the month $(\widetilde{0})$ & $(0,0,1)$ \\
In about one week $(\widetilde{1})$ & $(0,1,2)$ \\
In about two weeks $(\widetilde{2})$ & $(1,2,3)$ \\
In about three weeks $(\widetilde{3})$ & $(2,3,4)$ \\
At the end of the month $(\widetilde{4})$ & $(3,4,4)$ \\
\hline
\end{tabular}

Unless any risks occur during the month, the monthly supply capacity will reach this point

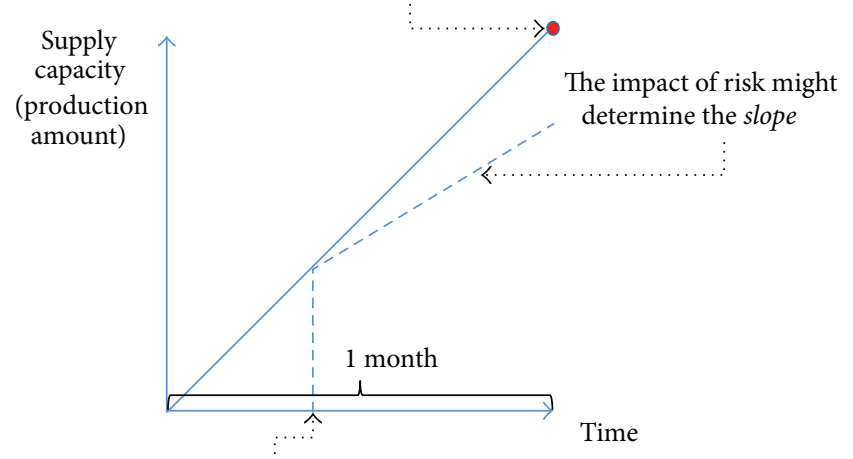

The timing of risk might determine the inflection point

FIGURE 1: Estimating the supply capacity using both the timing and the impact of risk.

where $L^{T}=\min _{k}\left\{l_{k}^{T}\right\}, M^{T}=\sum_{k=1}^{K} m_{k}^{T} / K$, and $U^{T}=$ $\max _{k}\left\{u_{k}^{T}\right\}$.

Once the timing of risk is determined as the integrated fuzzy number $(\widetilde{T})$, the impact of risk also needs to be determined to estimate supply capacity. Similar to the case of risk timing determination, an expert could answer a question such as how big the risk will become using the linguistic expressions in Table 2. As for the timing of the risk, if the expert expects no impact from the risk, he or she simply can answer " 4 " to this question.

The membership function corresponding to Table 2 is shown in Figure 3.

In Table 2, unlike the conventional representation, the larger impact of risk has the smaller triangular fuzzy number. This is because the larger impact of risk reduces the slope of the supply capacity line (e.g., the line could stop owing to the large impact of risk). In Figure 3, the horizontal scale of the membership function can be either lengthened or shortened according to the timing of risk, even if the determinant of the slope is always set from 0 to 4 in our research. For example, if the risk occurs at the beginning of the month, the horizontal scale of the membership function should be longer than it is when the risk occurs later. However, the horizontal scale might be substantially shorter when the risk occurs at the end of month. In short, when estimating supply capacity, the impact of risk can be considered after the timing of risk is discussed among supply chain experts who will give their
TABLE 2: Fuzzy numbers regarding the impact of risk.

\begin{tabular}{lc}
\hline Linguistic expression of opinion & Triangular fuzzy number \\
\hline Extremely large $(\widetilde{E})$ & $(0,0,1)$ \\
Large $(\widetilde{L})$ & $(0,1,2)$ \\
Medium $(\widetilde{M})$ & $(1,2,3)$ \\
Small $(\widetilde{S})$ & $(2,3,4)$ \\
None $(\widetilde{N})$ & $(3,4,4)$ \\
\hline
\end{tabular}
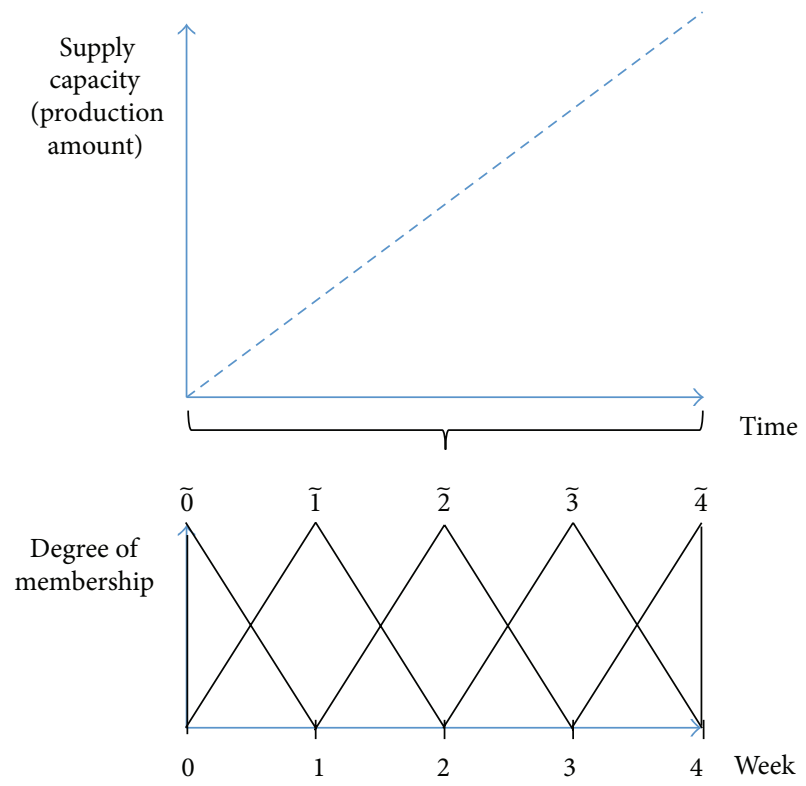

FIGURE 2: Membership function regarding the timing of risk.

opinions on the upcoming potential risks. Figure 4 shows two different cases of determining the impact of risk.

As in the determination of $\widetilde{T}$, the integrated fuzzy number representing the timing of risk, $K$ different opinions on the impact of risk need to be merged. Let us assume that $\widetilde{S}$ is the integrated fuzzy number indicating the impact of risk, $l_{k}^{S}$ is the lower value of the triangular fuzzy number representing the impact of risk, $m_{k}^{S}$ is the mean value of the triangular fuzzy number representing the impact of risk, and $u_{k}^{S}$ is the upper value of the triangular fuzzy number representing the impact of risk. Then, $\widetilde{S}$ can be obtained from the following equation:

$$
\widetilde{S}=\left(L^{S}, M^{S}, U^{S}\right),
$$

where $L^{S}=\min _{k}\left\{l_{k}^{S}\right\}, M^{S}=\sum_{k=1}^{K} m_{k}^{S} / K$, and $U^{S}=\max _{k}\left\{u_{k}^{S}\right\}$.

Once both $\widetilde{T}$ and $\widetilde{S}$ are determined, we need to estimate the supply capacity of foreign suppliers, $C_{f t}$. The first step is to convert the fuzzy numbers into crisp numbers.

To do this, we need to characterize the given triangular fuzzy numbers by defining the interval of confidence level $\alpha$ as follows:

$$
\widetilde{a}^{\alpha}=\left[l^{\alpha}, u^{\alpha}\right]=[(m-l) \alpha+l, u-(u-m) \alpha]
$$

$\forall \alpha \in[0,1]$. 


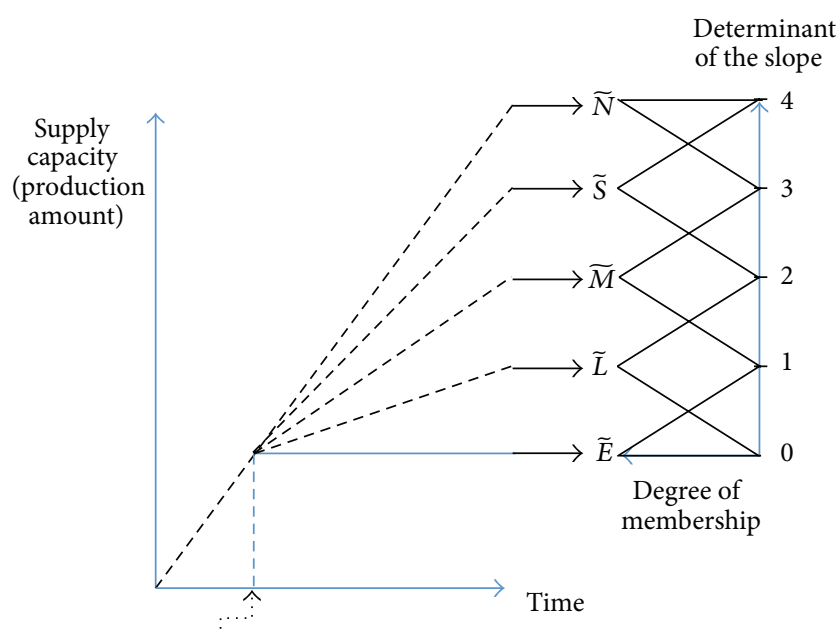

If the risk occurs here

FIGURE 3: Membership function regarding the impact of risk.

For example, when the interval of confidence level is set to $\alpha, \widetilde{T}$ can be characterized as follows:

$$
\begin{aligned}
\widetilde{T}^{\alpha}= & {\left[l(\widetilde{T})^{\alpha}, u(\widetilde{T})^{\alpha}\right] } \\
= & {\left[\left(M^{T}-L^{T}\right) \alpha+L^{T}, U^{T}-\left(U^{T}-M^{T}\right) \alpha\right] } \\
& \forall \alpha \in[0,1] .
\end{aligned}
$$

This is an $\alpha$-cut method known to incorporate the supply chain experts' confidence in their opinions [35]. As shown in (23), the $\alpha$-cut method yields an interval set of values from a fuzzy number: the lower and upper limits of the fuzzy numbers with respect to the $\alpha$-cut. In general, the value of $\alpha$ is set between 0 and 1 . The degree of uncertainty is greatest and the degree of confidence is least when $\alpha=$ 0 . The degree of uncertainty decreases and the degree of confidence increases as $\alpha$ approaches 1 [36]. Next, we convert the triangular fuzzy numbers into crisp numbers by applying the index of optimism, $\mu$. A larger value of $\mu$ indicates a higher degree of optimism. The index of optimism is a linear convex combination as follows [37]:

$$
\begin{array}{ll}
C(\widetilde{T})=\mu \times u(\widetilde{T})^{\alpha}+(1-\mu) \times l(\widetilde{T})^{\alpha} & \forall \mu \in[0,1], \\
C(\widetilde{S})=\mu \times u(\widetilde{S})^{\alpha}+(1-\mu) \times l(\widetilde{S})^{\alpha} \quad \forall \mu \in[0,1],
\end{array}
$$

where $C(\widetilde{T})$ and $C(\widetilde{S})$ are crisp values corresponding to $\widetilde{T}$ and $\widetilde{S}$, respectively, considering the index of optimism $\mu$ and the confidence level $\alpha$ of (23).

The next step for estimating $C_{f t}$ is to generate the final estimated value using $C(\widetilde{T})$ and $C(\widetilde{S})$. As shown in Figure 5 , $C_{f t}$ can be calculated as follows:

$$
C_{f t}=\frac{C(\widetilde{T})}{4} C_{f t}^{O}+\left(C_{f t}^{O}-\frac{C(\widetilde{T})}{4} C_{f t}^{O}\right) \frac{C(\widetilde{S})}{4},
$$

where $C_{f t}^{O}$ is the possible supply capacity when there is no risk during the month (i.e., four weeks).
3.3. Solution Procedure. If the problem size is not too large, the GPP model can be solved using a conventional branchand-bound algorithm embedded in the commercial optimization software such as IBM ILOG CPLEX and LINGO. Actually, in our case study, the computational time for solving the GPP model was under one second. However, when the problem size is too large to use the commercial optimization software due to the computational burden, we need to consider adopting heuristic approaches which can give a good solution within a reasonable time. In this research, we adopt a genetic algorithm based integrated production planning (GA-IPP) approach developed and validated by Jung et al. [38]. The detailed explanation on the GA-IPP can be found from Jung et al. [38].

\section{Case Study}

To demonstrate the applicability of the proposed method and model, we present the case study of a footwear supply chain. We focus in particular on the shoemaking process. We assume that the shoes are composed of three major components: insole, laces, and body. The shoe body includes all components (like the toe cap and heel) except the insole and laces. This case study is based on a real world company (hereafter A Company) in Korea. To maintain confidentiality, all parameter values in this case study are hypothetical, and the supply chain structure is compressed for the sake of simplicity. A Company produces three types of main products $(k=3)$ and has three main customers $(c=3)$ in the domestic and international markets. Furthermore, A Company has two overseas designated production facilities $(f=2)$ to produce the leather-based body and latex-based insole in Indonesia, and it has one shoemaking (i.e., assembly) line $(a=1)$ and two distribution centers $(d=2)$ in Korea. In other words, each overseas production facility produces either the body or the insole using various materials received from local suppliers. A Company can produce a final product by assembling multiple components (i.e., the body and the insole) received from those overseas production facilities. To simplify the case, it is assumed that no other parts or components are needed to produce the final product. Figure 6 shows supply chain of A Company.

The final products then move to the customers via distribution centers by two transport modes $(v=2)$ : air and marine. Furthermore, the basic time unit is a month consisting of four weeks, and the time period is set to be from September to December $2014(t=4)$. Since the supply risks in this research are assumed to be qualitative, such as social, political, and economic issues, the basic time unit of the GPP model is set to the large time bucket of the month. The production facilities in Indonesia had several important labor issues in late 2014. The minimum wage of Indonesian workers increased dramatically based on Indonesian government policy over the past several years. In 2013, the monthly minimum wage was set to about $\$ 230$, which was almost twice that of 2010 (i.e., \$126), despite a fair inflation rate (i.e., 5.3\% in 2013) [39]. Thus, A Company's management team is worried about the labor stability of their 




(a) When the risk occurs early

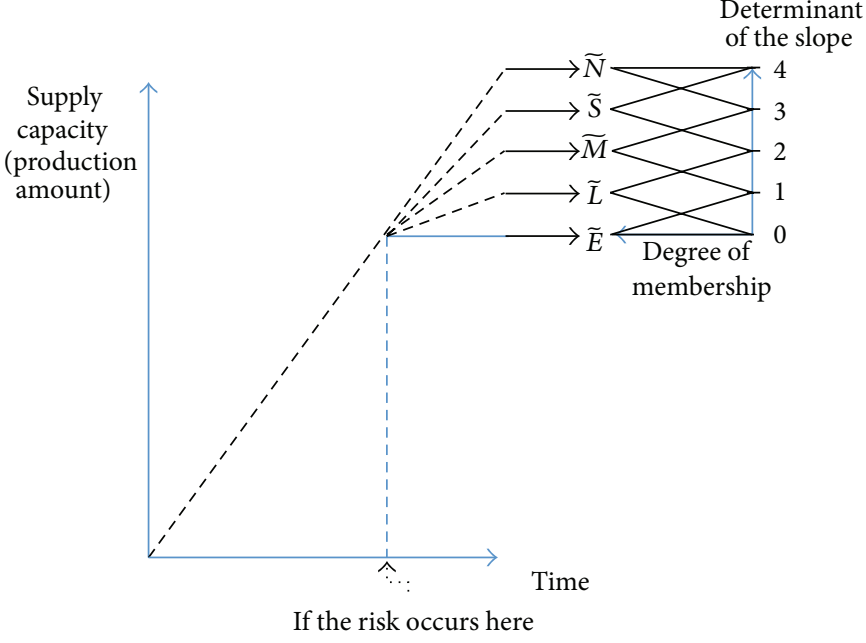

(b) When the risk occurs late

FIGURE 4: Two different membership functions regarding the impact of risk.



FIGURE 5: Estimating the final supply capacity.

production facilities and has hesitated to recruit new workers to increase supply capacity. In addition, the turnover rate has been relatively high in the fall for the past couple of years, and the annual wage negotiation, which can lead to labor strikes, was due to occur in December. In short, A Company's management team needed to estimate the supply capacity of their production facilities in Indonesia carefully considering the above-mentioned local risks. To generate the supply capacity of the two production facilities in Indonesia for the upcoming quarter, we applied the supply capacity estimation process from Section 3.2 with the help of three local experts who have worked in these production facilities for more than seven years.

Table 3 shows the step-by-step results of the supply capacity estimation process for A Company. First, the ideal supply capacities obtained by assuming the perfect and ideal working conditions are shown in Table 3(a). Next, we asked the three experts to judge the timing of risk (i.e., when the supply risk could occur during each quarter) using the linguistic expressions in Table 1 (Table 3(b)). We also asked the three experts to answer the question about the impact of risk (i.e., how big that risk would become) using the linguistic expressions in Table 2 (Table 3(c)). For reference, because the three experts expected no risk in November, we need not consider the impact of any risks for November. Once the judgments on both the timing and impact of risk are prepared, we can determine their integrated fuzzy numbers using (21) and (22), respectively (Table 3(d)). The integrated fuzzy numbers can be converted into crisp numbers using (23)-(25). In (23) and (24), the interval of confidence level $\alpha$ is set to 0.8 , while the index of optimism is set to 0.5 in (25) according to the previous research [40]. The converted crisp numbers for both timing and impact of risk are shown in Table 3(e). Finally, using the crisp numbers and (26), we can determine the estimated supply capacities of two production facilities as shown in Table 3(f).

Using the estimated supply capacities and any other required data, we ran the GPP model. Since the GPP model is a MILP model, we used the MILP module of Gurobi 5.6.2, the commercial optimization software, along with Python programming language, which handles data processing before and after running the GPP model. After running the GPP model, we obtained the results for the sales, cost, and total profit during the planning periods as shown in Table 4.

Additionally, we tried to compare the proposed supply capacity estimation to the current practice in A Company. The management team of A Company currently employs a simple estimation rule called SER. The basic idea is as follows:

(i) Unless any risks are expected for any given planning period, the supply capacities are set to $90 \%$ of the ideal ones shown in Table 3(a).

(ii) If any risks which can affect production are expected for any given planning period, the supply capacities are set to $60 \%$ of the ideal ones. 


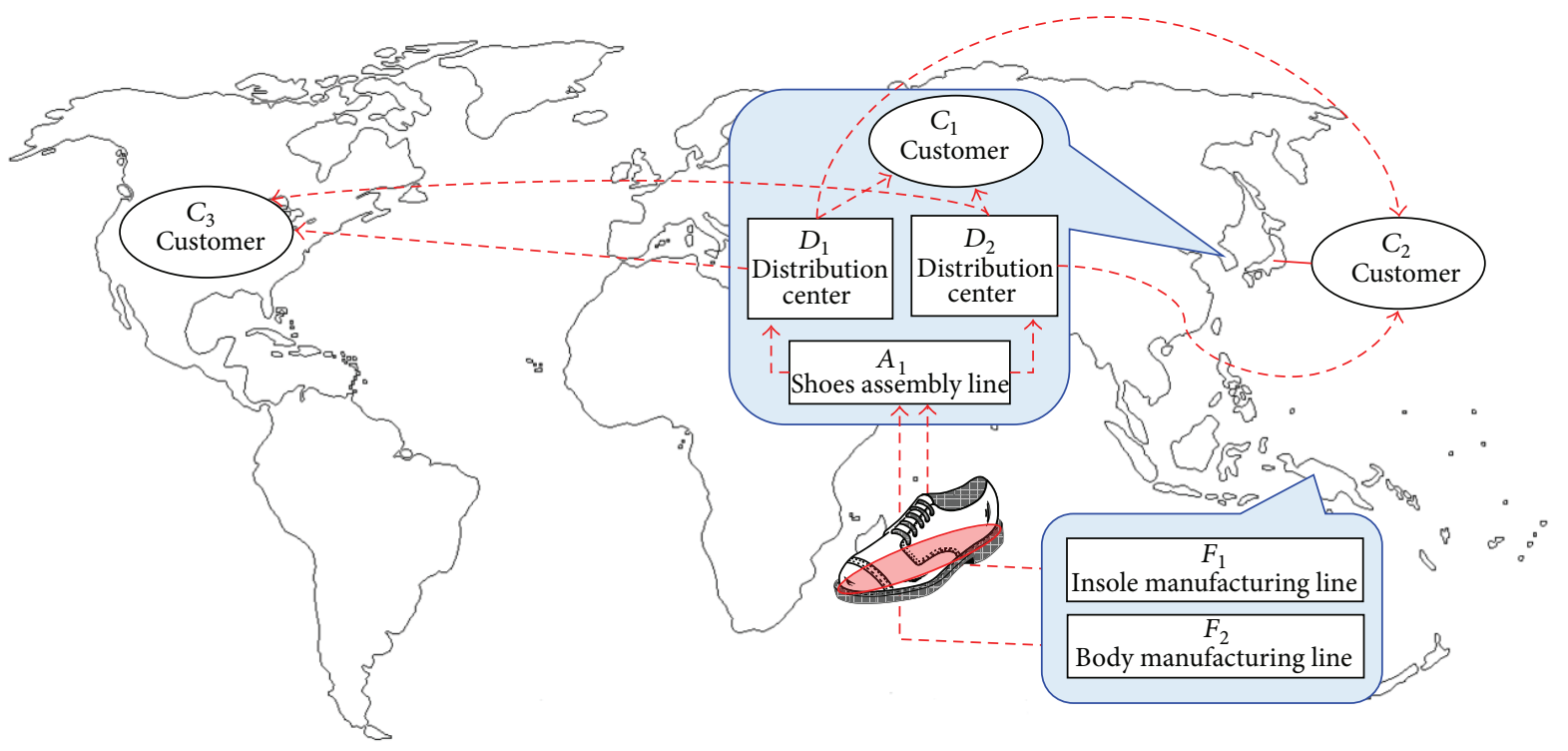

FIgURE 6: The supply chain of A Company.

In this case study, the management team of A Company expected the labor risks in September, October, and December. Thus, the estimated supply capacities for two production facilities using SER are as shown in Table 5.

After running the GPP model with two different settings for the supply capacity, SER and the proposed one, we found that there are no differences in sales between two approaches during the time periods. This was because the sales were pegged to the given demand instead of the supply capacity. Fulfilling the whole demand generates the maximum sales regardless of the settings of the supply capacity unless the supply capacities are set to be much lower than the demand. In other words, with the enough total supply capacity, A Company can provide all the ordered products without shortages regardless of the supply capacity setting. As for the cost, SER showed better results (i.e., lower cost) in comparison with the proposed approach. However, these results were the calculated (or expected) cost only from the generated plan, and thus we needed to compare the costs with the real one which can be checked in four months (i.e., planning periods). With the help of the management team of A Company, we could calculate the approximate percentage gap between the costs obtained from both SER and the proposed approach and the real cost. While there was a 1.07 percentage point gap between the planned cost from the proposed approach and the real cost, SER showed a 2.62 percentage point gap. Table 6 shows those comparison results.

Of course, supply capacity estimation can be considered as another forecasting in the firms, and thus it could be wrong due to its intrinsic limit. However, instead of adopting any simple estimation rule, a more systematic and reasonable way can be adopted to estimate both the timing and the impact of the expected upcoming risks. Based on an interview with the A Company management team, the proposed estimation process looks viable.
Some important findings and managerial implications can be summarized again as follows:

(i) Comparing with the ideal supply capacity used in the previous planning, the estimated supply capacity might give a more reasonable plan which considers the upcoming potential risks. Based on an interview with the management team of A Company, the proposed estimation process generated a better plan in terms of the difference between the expected cost and the real cost. When using SER, the expected total cost seems to be lower at first, but the real cost increased in four months (i.e., planning horizon) due to the unexpected events.

(ii) Since the existing global production planning approach has a relatively little interest in estimating the supply capacity, the decision-maker has a tendency to set the supply capacity to the ideal value without considering risks. But, in the proposed supply capacity estimation process, both the timing and impact of the potential risks can be considered together to set the supply capacity which will be used in global production planning.

With the proposed approach, the decision-makers and the related practitioners will be able to generate a more reliable global production plan carefully considering the supply risk of overseas manufacturing sites.

\section{Conclusions}

In this paper, we proposed a new GPP process considering the supply risk of overseas manufacturing sites. As offshoring has prevailed in most global manufacturers to increase cost competitiveness, accurate GPP has become one of the most 
TABLE 3: Step-by-step results of supply capacity estimation process.

(a) Ideal supply capacities without considering any risks

\begin{tabular}{lcccc} 
& September $(t=1)$ & October $(t=2)$ & November $(t=3)$ & December $(t=4)$ \\
\hline Production facility \#1 & 1160 & 1181 & 1346 & 1400 \\
Production facility \#2 & 1602 & 1305 & 1564 & 1249 \\
\hline
\end{tabular}

(b) Judgments of the experts on the timing of risk

\begin{tabular}{lcccc} 
& September $(t=1)$ & October $(t=2)$ & November $(t=3)$ & December $(t=4)$ \\
\hline Expert 1 & At the beginning of the month & In about three weeks & No risk & At the beginning of the month \\
Expert 2 & In about one week & In about three weeks & No risk & In about one week \\
Expert 3 & In about one week & In about two weeks & No risk & In about two weeks \\
\hline
\end{tabular}

Expert 3

(c) Judgments of the experts on the impact of risk

\begin{tabular}{lcccc} 
& September $(t=1)$ & October $(t=2)$ & November $(t=3)$ & December $(t=4)$ \\
\hline Expert 1 & Large & Small & No risk & Extremely large \\
Expert 2 & Medium & None & No risk & Large \\
Expert 3 & Large & Small & No risk & Extremely large \\
\hline
\end{tabular}

Expert 3

(d) The integrated fuzzy numbers for both the timing and impact of risk

\begin{tabular}{lcccc} 
& September $(t=1)$ & October $(t=2)$ & November $(t=3)$ & December $(t=4)$ \\
\hline Timing of risk & $(0,0.67,2)$ & $(1,2.67,4)$ & $(4,4,4)$ & $(0,1,3)$ \\
Impact of risk & $(0,1.33,3)$ & $(2,3.33,4)$ & $(4,4,4)$ & $(0,0.33,2)$ \\
\hline
\end{tabular}

Impact of risk

(e) The converted crisp numbers for both timing and impact of risk

\begin{tabular}{lcccc} 
& September $(t=1)$ & October $(t=2)$ & November $(t=3)$ & December $(t=4)$ \\
\hline Timing of risk & 0.733 & 2.633 & 4 & 1.1 \\
Impact of risk & 1.366 & 3.266 & 4 & 0.466 \\
\hline
\end{tabular}

\begin{tabular}{lcccc}
\hline & \multicolumn{2}{c}{$(\mathrm{f})$ The final estimated supply capacities } & \\
& September $(t=1)$ & October $(t=2)$ & November $(t=3)$ & December $(t=4)$ \\
\hline Production facility \#1 & 536 & 1107 & 1346 & 503 \\
Production facility \#2 & 741 & 1223 & 1564 & 449 \\
\hline
\end{tabular}

TABLE 4: The results of the GPP model running.

\begin{tabular}{lcccccc}
\hline & September $(t=1)$ & October $(t=2)$ & November $(t=3)$ & December $(t=4)$ & Sum & Total profit \\
\hline Sales & 135000 & 122250 & 227000 & 405000 & 889250 \\
Cost & 40512 & 41781 & 63802 & 68617 & 214711 & 674539 \\
\hline
\end{tabular}

Unit: USD.

TABLE 5: The estimated supply capacities using SER.

\begin{tabular}{lcccc}
\hline & September $(t=1)$ & October $(t=2)$ & November $(t=3)$ & December $(t=4)$ \\
\hline Production facility \#1 & 696 & 709 & 1211 & 840 \\
Production facility \#2 & 961 & 783 & 1408 & 749 \\
\hline
\end{tabular}

TABLE 6: Comparison between SER and the proposed process: gap from the real cost.

\begin{tabular}{lccccc}
\hline & September $(t=1)$ & October $(t=2)$ & November $(t=3)$ & December $(t=4)$ & Total cost gap \\
\hline SER & 5.92 & 0.55 & 0.71 & 6.45 & 2.62 \\
The proposed process & 1.86 & 0.78 & 1.28 & 4.90 & 1.07 \\
\hline
\end{tabular}

Unit: percentage.

important business activities. The accuracy of the plan might affect all following business activities such as human resource management and third-party logistics contracts. Thus, many global manufacturers are developing more systematic and accurate GPP processes. In this regard, firms need to consider how to reflect various risks originating from foreign government policies, regulations, and labor stability in GPP. In particular, the supply capacities of overseas manufacturing facilities should be determined by predicting the timing and impact of upcoming risks. To do this, we developed 
a fuzzy theory-based supply capacity estimation process that can systematically merge expert judgments of risks into an integrated value. Furthermore, we checked the applicability of the proposed process using a real world case study. From the case study, the proposed process seems to be viable and to be a good alternative to estimate the supply capacity considering the timing and impact of the risk.

In terms of future research directions, we need to perform long-term validation of the proposed process with real data from A Company. We are currently contacting practitioners at A Company to perform such validation. Additionally, some other cases with real data should be considered and validated in the near future to ensure that the proposed process is robust and reliable in any real world situations. Now, we are actively looking for real world partners (i.e., any manufacturing firms) to access real data and to validate our proposed process again.

Furthermore, the proposed process can be extended by considering other risk types such as demand and transportation risks. Although we considered the supply risks in this paper, there are various risks in the supply chain. In order to generate a more accurate global production plan, supply, demand, and transportation risks should be estimated in advance and reflected on the final production and distribution plan. Additionally, some risks might be interrelated from each other. For example, supply risks could lead to the transportation risks because supply risks (e.g., production delay or stoppage) could increase transportation uncertainty. Thus, we need to check the relationship among supply chain risks, and the impact of risks should be strengthened or weakened considering this relationship appropriately. In other words, since some risks might come together with intensity or other risks might occur independently, we need to reflect each case on the plan differently. Finally, some other fuzzy numbers can be considered to estimate supply capacities better than the linear triangular fuzzy numbers. Because the main contribution of this paper is not to compare various fuzzy numbers but to provide the systematic approach for quantifying the intuitive and subjective opinion of experts by considering two factors, the timing and the impact of the potential risks, we left comparison among various fuzzy numbers to the future research topic. We will adopt other fuzzy numbers to estimate supply capacity considering the opinion of the experts and will validate their performance in the near future.

\section{Conflict of Interests}

The authors declare that there is no conflict of interests regarding the publication of this paper.

\section{Acknowledgment}

This research was supported by Basic Science Research Program through the National Research Foundation of Korea (NRF) funded by the Ministry of Education, Science and Technology (NRF-2012R1A1A1011396).

\section{References}

[1] P. Tsiakis and L. G. Papageorgiou, "Optimal production allocation and distribution supply chain networks," International Journal of Production Economics, vol. 111, no. 2, pp. 468-483, 2008.

[2] S. Liu and L. G. Papageorgiou, "Multiobjective optimisation of production, distribution and capacity planning of global supply chains in the process industry," Omega, vol. 41, no. 2, pp. 369382, 2013.

[3] C. Martínez-Costa, M. Mas-Machuca, E. Benedito, and A. Corominas, "A review of mathematical programming models for strategic capacity planning in manufacturing," International Journal of Production Economics, vol. 153, pp. 66-85, 2014.

[4] G. Lanza and R. Moser, "Strategic planning of global changeable production networks," Procedia CIRP, vol. 3, pp. 257-262, 2012.

[5] N. K. Shah and M. G. Ierapetritou, "Integrated production planning and scheduling optimization of multisite, multiproduct process industry," Computers and Chemical Engineering, vol. 37, pp. 214-226, 2012.

[6] M. Díaz-Madroñero, D. Peidro, and J. Mula, "A review of tactical optimization models for integrated production and transport routing planning decisions," Computers \& Industrial Engineering, 2015.

[7] A. Jabbarzadeh, B. Fahimnia, and J. Sheu, "An enhanced robustness approach for managing supply and demand uncertainties," International Journal of Production Economics, 2015.

[8] F. Sahling and A. Kayser, "Strategic supply network planning with vendor selection under consideration of risk and demand uncertainty," Omega, 2015.

[9] U. Juttner, H. Peck, and M. Christopher, "Supply chain risk management: outlining an agenda for future research," International Journal of Logistics Research and Applications, vol. 6, no. 4, pp. 197-210, 2003.

[10] I. Manuj and T. J. Mentzer, "Global supply chain risk management," Journal of Business Logistics, vol. 29, no. 1, pp. 133-155, 2008.

[11] R. Narasimhan and S. Talluri, "Perspectives on risk management in supply chains," Journal of Operations Management, vol. 27, no. 2, pp. 114-118, 2009.

[12] S. Y. Ponomarov and M. C. Holcomb, "Understanding the concept of supply chain resilience," The International Journal of Logistics Management, vol. 20, no. 1, pp. 124-143, 2009.

[13] C. S. Tang, "Perspectives in supply chain risk management," International Journal of Production Economics, vol. 103, no. 2, pp. 451-488, 2006.

[14] B. Ritchie and C. Brindley, "Supply chain risk management and performance: a guiding framework for future development," International Journal of Operations \& Production Management, vol. 27, no. 3, pp. 303-322, 2007.

[15] C. Tang and B. Tomlin, "The power of flexibility for mitigating supply chain risks," International Journal of Production Economics, vol. 116, no. 1, pp. 12-27, 2008.

[16] S. Chopra and M. S. Sodhi, "Managing risk to avoid: supplychain breakdown," MIT Sloan Management Review, vol. 46, no. 1, pp. 53-61, 2004.

[17] A. Oke and M. Gopalakrishnan, "Managing disruptions in supply chains: a case study of a retail supply chain," International Journal of Production Economics, vol. 118, no. 1, pp. 168-174, 2009. 
[18] I. Heckmann, T. Comes, and S. Nickel, "A critical review on supply chain risk-definition, measure, and modeling," Omega, vol. 52, pp. 119-132, 2015.

[19] S. C. Ellis, R. M. Henry, and J. Shockley, "Buyer perceptions of supply disruption risk: a behavioral view and empirical assessment," Journal of Operations Management, vol. 28, no. 1, pp. 34-46, 2010.

[20] L. F. Escudero and P. V. Kamesam, "On solving stochastic production planning problems via scenario modelling," Top, vol. 3, no. 1, pp. 69-95, 1995.

[21] S. C. H. Leung, S. O. S. Tsang, W. L. Ng, and Y. Wu, "A robust optimization model for multi-site production planning problem in an uncertain environment," European Journal of Operational Research, vol. 181, no. 1, pp. 224-238, 2007.

[22] X. Zhang, M. Prajapati, and E. Peden, "A stochastic production planning model under uncertain seasonal demand and market growth," International Journal of Production Research, vol. 49, no. 7, pp. 1957-1975, 2011.

[23] S. Karabuk, "Production planning under uncertainty in textile manufacturing," Journal of the Operational Research Society, vol. 59, no. 4, pp. 510-520, 2008.

[24] D. Peidro, J. Mula, R. Poler, and F.-C. Lario, "Quantitative models for supply chain planning under uncertainty: a review," International Journal of Advanced Manufacturing Technology, vol. 43, no. 3-4, pp. 400-420, 2009.

[25] S. A. Torabi and E. Hassini, "Multi-site production planning integrating procurement and distribution plans in multiechelon supply chains: an interactive fuzzy goal programming approach," International Journal of Production Research, vol. 47, no. 19, pp. 5475-5499, 2009.

[26] H. Jung, "An available-to-promise process considering production and transportation uncertainties and multiple performance measures," International Journal of Production Research, vol. 50, no. 7, pp. 1780-1798, 2012.

[27] J. Wang and Y.-F. Shu, "Fuzzy decision modeling for supply chain management," Fuzzy Sets and Systems, vol. 150, no. 1, pp. 107-127, 2005.

[28] M. S. Pishvaee and S. A. Torabi, "A possibilistic programming approach for closed-loop supply chain network design under uncertainty," Fuzzy Sets and Systems, vol. 161, no. 20, pp. 26682683, 2010.

[29] T.-F. Liang, "Application of fuzzy sets to manufacturing/distribution planning decisions in supply chains," Information Sciences, vol. 181, no. 4, pp. 842-854, 2011.

[30] A. F. Guneri, A. Yucel, and G. Ayyildiz, "An integrated fuzzylp approach for a supplier selection problem in supply chain management," Expert Systems with Applications, vol. 36, no. 5, pp. 9223-9228, 2009.

[31] A. H. I. Lee, H.-Y. Kang, and W.-P. Wang, "Analysis of priority mix planning for the fabrication of semiconductors under uncertainty", The International Journal of Advanced Manufacturing Technology, vol. 28, no. 3-4, pp. 351-361, 2006.

[32] H. Deng, "Comparing and ranking fuzzy numbers using ideal solutions," Applied Mathematical Modelling, vol. 38, no. 5-6, pp. 1638-1646, 2014.

[33] C.-B. Cheng, "Group opinion aggregationbased on a grading process: a method for constructing triangular fuzzy numbers," Computers and Mathematics with Applications, vol. 48, no. 10-11, pp. 1619-1632, 2004.

[34] D.-F. Li, "A fast approach to compute fuzzy values of matrix games with payoffs of triangular fuzzy numbers," European
Journal of Operational Research, vol. 223, no. 2, pp. 421-429, 2012.

[35] Z. Güngör, G. Serhadlıŏlu, and S. E. Kesen, "A fuzzy AHP approach to personnel selection problem," Applied Soft Computing, vol. 9, no. 2, pp. 641-646, 2009.

[36] N.-F. Pan, "Fuzzy AHP approach for selecting the suitable bridge construction method," Automation in Construction, vol. 17, no. 8, pp. 958-965, 2008.

[37] A. R. Lee, Application of modified fuzzy AHP method to analyze bolting sequence of structural joints [Ph.D. thesis], Lehigh University, Bethlehem, $\mathrm{Pa}, \mathrm{USA}, 1995$.

[38] H. Jung, I. Song, and B. Jeong, "Genetic algorithm-based integrated production planning considering manufacturing partners," The International Journal of Advanced Manufacturing Technology, vol. 32, no. 5-6, pp. 547-556, 2007.

[39] K. Vaswani, "Indonesia's wage wars," BBC News, 2013, http:// www.bbc.com/news/business-21840416.

[40] H. Jung, "A fuzzy AHP-GP approach for integrated productionplanning considering manufacturing partners," Expert Systems with Applications, vol. 38, no. 5, pp. 5833-5840, 2011. 


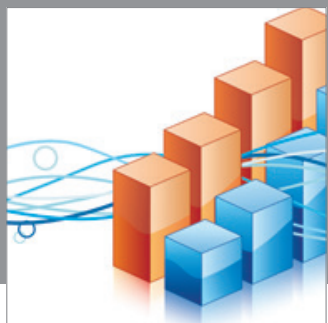

Advances in

Operations Research

mansans

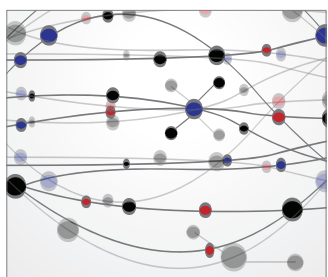

The Scientific World Journal
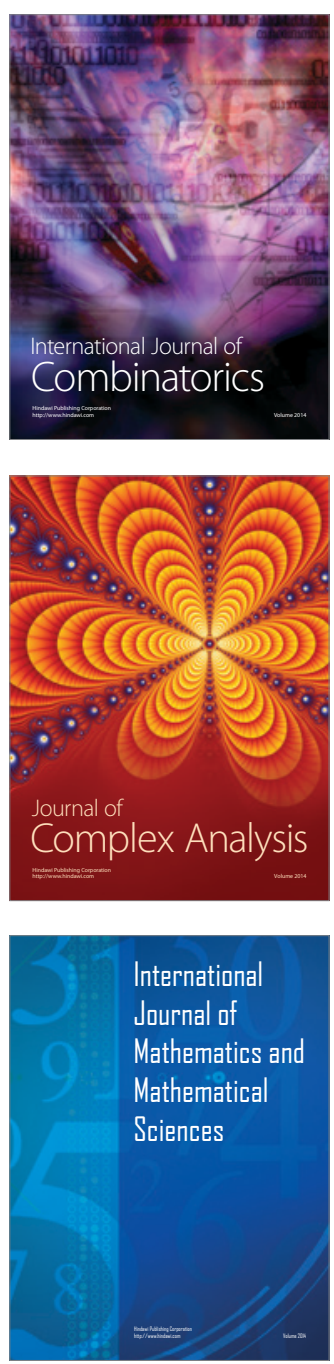
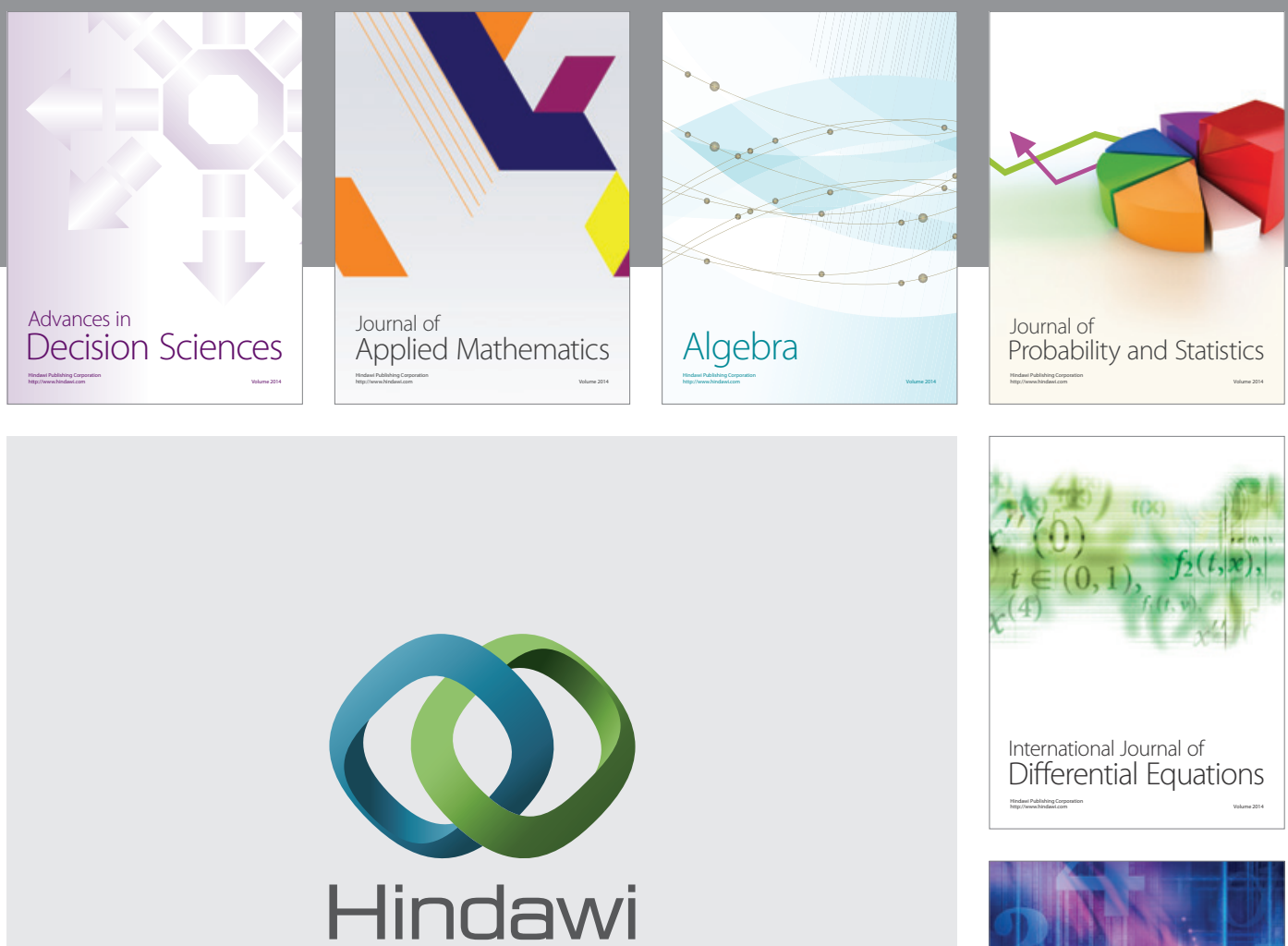

Submit your manuscripts at http://www.hindawi.com
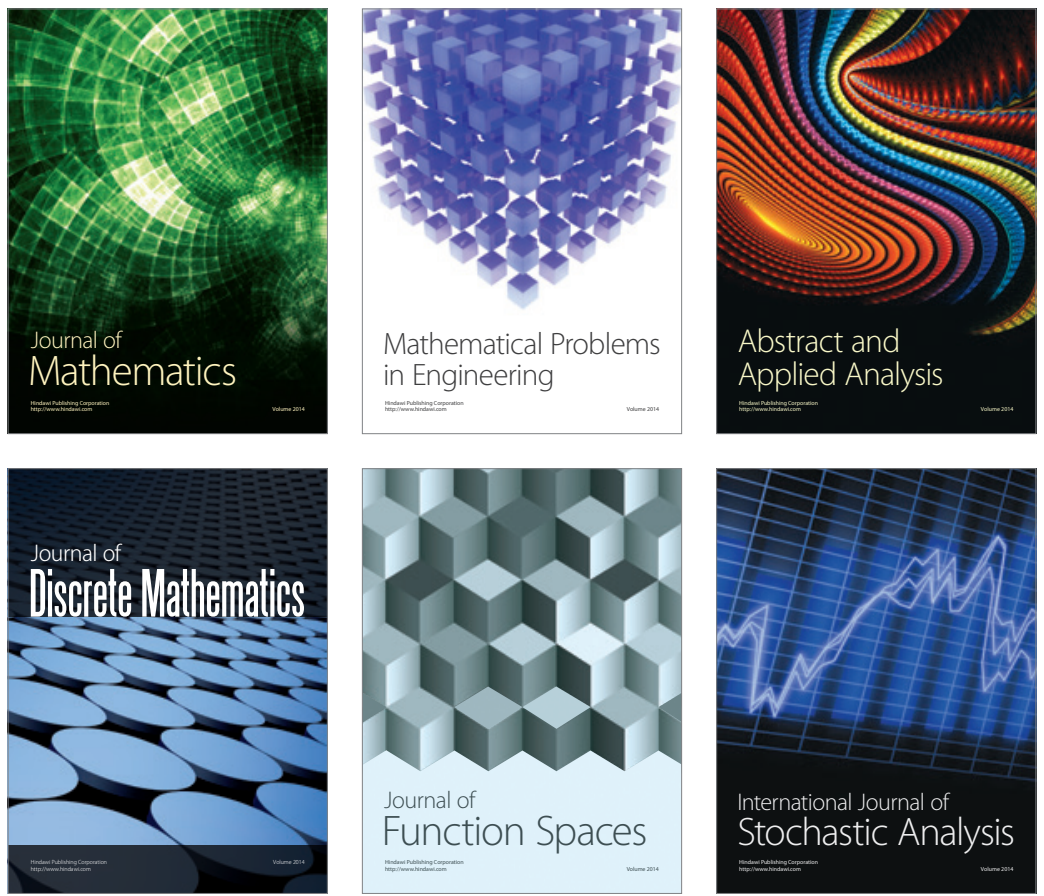

Journal of

Function Spaces

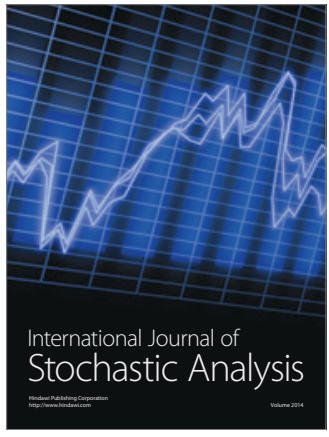

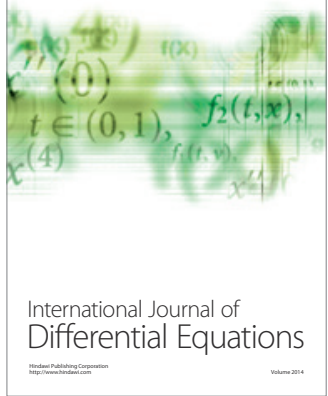
